University of Washington Tacoma

UW Tacoma Digital Commons

2-1-2019

\title{
Federal Weatherization and Health Education Team Up: Process Evaluation of a New Strategy to Improve Health Equity for People With Asthma and Chronic Obstructive Pulmonary Disease
}

\author{
Rachael De Souza \\ Robin Evans-Agnew \\ University of Washington Tacoma, robagnew@u.washington.edu \\ Christine Espina \\ Western Washington University
}

Follow this and additional works at: https://digitalcommons.tacoma.uw.edu/nursing_pub

Part of the Nursing Commons

\section{Recommended Citation}

De Souza, Rachael; Evans-Agnew, Robin; and Espina, Christine, "Federal Weatherization and Health Education Team Up: Process Evaluation of a New Strategy to Improve Health Equity for People With Asthma and Chronic Obstructive Pulmonary Disease" (2019). Nursing \& Healthcare Leadership Publications. 162.

https://digitalcommons.tacoma.uw.edu/nursing_pub/162

This Article is brought to you for free and open access by the Nursing \& Healthcare Leadership at UW Tacoma Digital Commons. It has been accepted for inclusion in Nursing \& Healthcare Leadership Publications by an authorized administrator of UW Tacoma Digital Commons. 
Federal weatherization and health education team up: Process evaluation of a new strategy to improve health equity for people with asthma and COPD

Rachael De Souza, MN, MPH, RN

unaffiliated

Robin Evans-Agnew, PhD, RN,

Assistant Professor

Nursing and Healthcare Leadership

University of Washington Tacoma

Christine Espina, DNP, MN, BSN, RN

Assistant professor

Health and Community Studies

Acknowledgements: We wish to acknowledge the collaboration of all members of the PCHS/TPCHD Weatherization Plus Health team: Teri Allen, Elodia Andres Cornelio, Jennifer Bauer, Rachael De Souza, Michael Johnson, RaKisha McCain, Shelley Minetti, Judy Olsen, Brian Sarensen, and Eric Seeman. 


\begin{abstract}
Unhealthy housing is a major cause of respiratory health inequity. In-home health education improves health equity for low-income and minority populations with asthma and COPD. Yet outcomes of educational interventions are limited by poor housing conditions. Federal weatherization programs represent a promising source of funding for home repairs. Innovative legislation in Washington State integrates the two interventions as "Weatherization Plus Health," offering environmental health education in partnership with the Weatherization Assistance Program for low-income families with respiratory disease. This practice brief describes process evaluation results of a Weatherization Plus Health program in Pierce County, WA. Evaluation data was gathered via interviews with service providers and ethnographic observation. Workers report that the combined intervention expanded their understanding of their practice, their skills, and their feelings of efficacy in meeting client needs. Integrating federally-funded home weatherization with health education shows promise for building public health system capacity and increasing health equity.
\end{abstract}

\title{
Key Words:
}

Healthy Housing, Asthma, COPD, Community Health Workers, Weatherization Assistance Program 


\section{Practice Brief Report}

Unhealthy housing is a major cause of respiratory health inequity. Living in a home with poor indoor air quality is strongly associated with increased asthma development and symptoms ${ }^{1}$, as well as increased chronic obstructive pulmonary disease (COPD) exacerbations. ${ }^{2}$ Older adults, children, and lowincome families are especially vulnerable. ${ }^{3,4}$ In order to improve health outcomes, people with asthma or COPD need healthier housing conditions and must be empowered to maintain a healthy home environment. ${ }^{5}$ The 2012 Coordinated Federal Action Plan to Reduce Racial and Ethnic Asthma Disparities called for increased integration between federal programs providing housing and other public health services for addressing asthma inequities. ${ }^{6}$ Such initiatives emphasize the urgent need for public health programmers to continue pursuing opportunities in health services integration. ${ }^{7}$

The Federal Weatherization Assistance Program (WAP) is an established safety-net program that has been providing insulation and heating systems for low-income families since $1973 .{ }^{8}$ While the original goal of WAP was to reduce fossil fuel consumption, WAP families with asthma experience fewer asthmarelated emergency room visits, hospitalizations, hospital readmissions and missed school/work days than before weatherizing their homes. ${ }^{9,10}$ Community Health Worker (CHW) Programs delivering inhome public health education for people with asthma and COPD have similarly led to fewer asthma- and COPD-related symptoms, activity limitations, and exacerbations requiring medical care. ${ }^{11,12}$ However, the benefits of $\mathrm{CHW}$ programs appear limited by their ability to provide trigger-reduction home improvements and repairs, such as improved ventilation and pest control. ${ }^{12}$ Combining WAP's home improvements with an education program teaching clients about respiratory disease and home environmental health could multiply the health benefits of both intervention strategies. ${ }^{13}$ This practice brief describes process outcomes from a novel combination of the WAP with an existing public health department $\mathrm{CHW}$ program using data collected during routine process improvement meetings and 
interviews with service providers. We first describe the program components and then summarize our findings.

\section{Program Description}

Weatherization Plus Health (known as $\mathrm{W}+\mathrm{H}$ ) is a new program with the goal of improving asthmaand COPD-related health outcomes for especially vulnerable families, by developing the shared capacity of two existing, publically funded programs. ${ }^{14}$ It is a partnership between Pierce County Human Services' WAP program, and Tacoma-Pierce County Health Department's asthma education program. Funded since June 2016 as part of a state legislative Healthy Housing Initiative ${ }^{15}$, the partnership provides home improvements and health education to households within Pierce County who meet federal requirements for WAP and also have a family member diagnosed with asthma or COPD.

The $\mathrm{W} x+\mathrm{H}$ program delivers traditional WAP home energy audit and energy efficiency improvements (such as insulation, air sealing, and heating system servicing), while adding healthfocused improvements such as bathroom and kitchen ventilation, roofing and plumbing repairs, and old carpet removal. The program provides clients with health promotion supplies including an allergycertified vacuum cleaner, heavy-duty walk off mat, furnace filters, hygrometer, carbon monoxide and smoke detectors, and non-toxic cleaning supplies. Clients receive personalized, in-depth health education from a CHW using motivational interviewing techniques. Education covers respiratory diagnoses, medication review, symptom management coaching, facilitated advocacy with medical providers, education on how to use and maintain all supplies provided by the program, and follow-up health education for one year. 
The $\mathrm{W} x+\mathrm{H}$ team consists of ten service providers. Three weatherization technicians conduct the energy audits, make home improvement recommendations, and facilitate contractor work in clients' homes. All technicians have Building Analyst, Energy Auditor, and Quality Control Inspector certifications, and two are certified Healthy Homes Specialists. Three CHWs provide the health education. Two CHWs have extensive lived experience and basic training in asthma education, and one has training in COPD education as a community health nurse. All CHWs have basic home environmental health training, and one has Master Home Environmentalist certification. Two CHWs provide English and Spanish services. Two office-based administrators facilitate client enrollment and scheduling, and two supervisors (one from each existing program) manage the team through monthly coordination meetings.

During its first year, $\mathrm{Wx}+\mathrm{H}$ reached 146 individuals living in 59 single-family homes. Of these individuals, 66 with asthma, 20 with COPD, and their family care givers, received personalized health education. On average per household, energy-efficiency-related home improvements cost $\$ 14900$, and health-related home improvements and supplies cost \$2 830 .

We collected evaluation data during from June 2016 through June 2017, via biweekly processimprovement meetings with the $\mathrm{WX}+\mathrm{H}$ team, as well as semi-structured interviews with each provider. Evaluation sought to identify $\mathrm{W} x+\mathrm{H}$ strengths and areas for improvement as perceived by the service providers in the initial year of the program.

\section{Findings}

$\mathrm{Wx}+\mathrm{H}$ provides valuable interventions 
Service providers note that $\mathrm{Wx}+\mathrm{H}$ adds value for clients, as exemplified by the improved air quality/ heating system component. During the $\mathrm{Wx}+\mathrm{H}$ initial energy audit, technicians use an AirAdvice monitor to measure not only temperature and humidity, like the traditional WAP audit, but also levels of volatile organic compounds (VOCs), particulate matter (PM 2.5), and carbon monoxide (CO), air-borne pollutants that can cause acute and chronic health problems. Technicians then install heat pumps enhanced with cold-plasma filters for heating and air quality control. The heat pump transfers heat between warm and cold areas rather than creating heat, making it a very energy efficient heating system. The cold-plasma filters remove VOCs and PM 2.5, improving indoor air quality. Additionally, CHWs provide health education on how to reduce PM2.5, VOC, and CO exposure through behavior changes, and supply families with simple air quality monitors for follow up monitoring.

CHWs see value in having tangible early results for sustained client motivation. Installing a more efficient heating system reduces clients' energy bills immediately, motivating them to address other areas of behavior change. Prior to $\mathrm{Wx}+\mathrm{H}, \mathrm{CHWs}$ recall visits to the same apartment complexes for the same mold problems adversely affecting different clients, and frustration in the inability to fix the root cause, which could be a leak or drainage problem. Providing structural improvements enhances the health education program and increases client empowerment and motivation.

Service providers develop new partnerships

Service providers report expanding their knowledge and abilities in $\mathrm{Wx}+\mathrm{H}$. By sharing scheduled visits, CHWs learn about structural problem mitigation, and technicians learn about health impacts of behaviors, leading to better-informed conversations with clients. Through partnership, service providers are exposed to the diverse programs of two separate agencies. This awareness enables providers to generate cross referrals across agencies, providing seamless services for clients. 
Technicians and CHWs report learning from the each other's approaches to service provision. Technicians note CHWs' strategies to engage clients, such as flexibility in scheduling appointments with working families and translating education materials for people of diverse backgrounds. As a result, technicians consider scheduling contracted work and technical inspections outside normal business hours. CHWs value technicians' expertise, dedication to create within-budget solutions for all families, and their flexibility to adjust to situation-specific construction projects.

Client recruitment and enrollment is challenging

$\mathrm{W} x+\mathrm{H}$ changes the way $\mathrm{CHWs}$ recruit clients. Previously, $\mathrm{CHWs}$ received client education referrals directly from medical clinics, making outreach efforts largely unnecessary. Eligibility did not require income qualifications or home ownership. Now, as part of a federally-funded program, CHWs must target home owners who meet income and immigration status qualifications. The new eligibility rules have proven confusing for medical clinics, slowing down existing referral streams. For apartment-homes, the process for weatherizing apartment buildings has proven so complex that the program failed to approve any apartment-dwellers in its first year. These requirements may further inhibit recruitment of low-income families with asthma.

WAP had to adjust its recruitment strategies. Previously, WAP received referrals through lowincome assistance programs, so enrollment specialists had no need or method to gather and record client health information. This led to erroneously identifying some clients as qualifying for $\mathrm{Wx}+\mathrm{H}$, while others were surely missed. Another missing link was storing and sharing protected client information between two separate agencies. The job of creating shared, secure databases fell largely on the enrollment specialists, who did so despite a lack of training and time budgeted for this work. Service providers report that learning these databases was time consuming and confusing. 
Service providers find $\mathrm{W} x+\mathrm{H}$ enrollment and construction timeline slow. Before, there was little delay between receiving a referral and completing an education home visit. This quick turnaround was useful in serving clients after an emergency or hospitalization, when clients can be especially sensitive to learning about their condition. However, $\mathrm{Wx}+\mathrm{H}^{\prime}$ s long enrollment process delays education delivery, which could decrease the impact of the educational intervention. Also, $\mathrm{Wx}+\mathrm{H}$ requires purchasing different supplies and developing relationships with new contractors, which has slowed the construction timeline.

\section{Conclusions}

The $\mathrm{W} x+\mathrm{H}$ program provides a valuable case study of integration of public health integration. ${ }^{7}$ All service providers report increased satisfaction with community engagement, and greater awareness of the challenges in aligning their programs to improve enrollment and inter-program referrals. The combination of service provider expertise improves service quality, fidelity to existing protocols, and sustainability. Adding health education to home improvement programs enhances its meaningfulness for clients, suggesting that such education may be useful for all clients, even those without respiratory diagnoses. Further examination of the health-related client outcomes of this innovative program, such as rates of emergency medical visits, hospitalizations, missed school and work-days, will be forthcoming.

This practice brief has described process evaluation outcomes for an innovative home weatherization and health education program. The evaluation highlights the strengths of such a program relating to improved service quality. Also noted are several challenges relating to recruitment and enrollment. Qualification requirements and health screening protocols could be simplified. The home visit protocol could be made more responsive to client requests. Establishing reliable suppliers for products and services would streamline the home improvement timeline. While these challenges are 
noted, the opportunities for improving the health of clients with chronic disease in their homes through such service integration is a promising advancement in public health integration. 


\section{Implications for Policy and Practice}

Inter-agency partnerships can enhance public health service programs that address inequities in chronic respiratory disease. The partnership described here between a federally-funded Weatherization Assistance Program and a county health department encountered a variety of successes and challenges in its integration of services.

- Seamless and timely access to solutions that address structural problems such as housing repairs may strengthen the effectiveness of respiratory health education efforts conducted by CHWs

- Identification of opportunities for positive health impacts in all policies can strengthen even non-health-focused programs for clients

- Inter-professional service delivery enhances each profession's knowledge of available resources, and allows for exchange of new skills and strategies to engage clients, enabling improved and more coordinated service delivery

- Developing a partnership between two existing programs requires more than combining the service product. New outreach, referral, documentation and follow-through processes must be thoughtfully planned

- To better serve vulnerable families, future integration efforts should work to reduce structural inequities in enrollment policies, such as reducing paperwork and documentation requirements for clients

- Programs targeting health inequities related to home environmental health must consider ways to expand their reach to rental housing 


\section{References}

1. Quansah R, Jaakkola MS, Hugg TT, Heikkinen SAM, Jaakkola JJK. Residential dampness and molds and the risk of developing asthma: A systematic review and meta-analysis. PloS ONE. 2012;7(11): e47526.

2. Rasmussen RV, Borchsenius L, Winslow JB, Pstergaard ER. Associations between housing conditions, smoking habits and ventilatory lung function in men with clean jobs. Scand J Resp Dis. 1978;59(5): 264-276.

3. Bashir SA. Home is where the harm is: Inadequate housing as a public health crisis. Amer J Public Health. 2002;92(5):733-738

4. Hood E. Dwelling disparities: How poor housing leads to poor health. Environ Health Perspect. 2005;113(5):A310-A317

5. Krieger J, Jacobs DE, Ashley PJ, Baeder A, Chew GL, Dearborn D, Hynes P, Miller D, Morley R, Rabito F, Zeldin DC. Housing interventions and control of asthma-related indoor biologic agents: A review of the evidence. J Public Health Manag Pract. 2010;16(5 Suppl):S11-20.

6. Coordinated federal action plan to reduce racial and ethnic asthma disparities. epa.gov. https://www.epa.gov/asthma/coordinated-federal-action-plan-reduce-racial-and-ethnicasthma-disparities. Published May 2012. Accessed October 1, 2017.

7. Wallace P, Barry A, Boufford J, et al. Committee on Integrating Primary Care and Public Health. Primary care and public health: Exploring integration to improve population health. Washington, DC: Institute of Medicine of the National Academies; 2012. 
8. About the weatherization assistance program. Office of Energy Efficiency and Renewable Energy. https://energy.gov/eere/wipo/about-weatherization-assistance-program-0. Accessed November 1, 2017.

9. Schweitzer M, Tonn B. Non-energy benefits of the US weatherization assistance program: A summary of their scope and magnitude. Applied Energy. 2003;76(4):321-335.

10. Tonn B, Carroll D, Pigg S, Blasnik M, Dalhoff G, Berger J, Rose E, Hawkins B, Eisenberg J, Ucar F, Bensch I, Cowan C. Weatherization works: Summary of findings from the retrospective evaluation of the US DOE's weatherization assistance program. Oak Ridge Nat Lab. 2014;338.

11. Casa A, Trooster T, Garcia-Aymerich J, Roca J, Hernández C, Alonso A, del Pozo F, de Toledo P, Antó JM, Rodíguez-Roisín R, Decramer M, members of the CHRONIC Project. Eur Respir J. 2006;28(1):123-130.

12. Postma J, Karr C, Kieckhefer G. Community health workers and environmental interventions for children with asthma: A systematic review. J Asthma. 2009;46(6):564-576.

13. Kuholski K, Tohn E, Morley R. Healthy energy-efficient housing: Using a one-touch approach to maximize public health, energy, and housing programs and policies. J Public Health Manag Pract. 2010;16(5 Suppl):S68-74.

14. Weatherization Plus Health $(\mathrm{W} x+\mathrm{H})$. Department of Commerce. http://www.commerce.wa.gov/growing-the-economy/energy/weatherization-and-energyefficiency/matchmaker/weatherization-plus-health-wxh/. Accessed November 1, 2017 
15. Washington State healthy housing initiative healthy housing strategic plan: An integrated approach to protecting and improving human and home environmental health. Olympia, WA: WA State Dept of Health; 2013. 